\title{
Fabrication of high-quality-factor photonic crystal microcavities in InAsP/InGaAsP membranes
}

\author{
Kartik Srinivasan, ${ }^{\text {a) }}$ Paul E. Barclay, and Oskar Painter \\ Department of Applied Physics, California Institute of Technology, Pasadena, California 91125 \\ Jianxin Chen and Alfred Y. Cho \\ Bell Laboratories, Lucent Technology, 600 Mountain Avenue, Murray Hill, New Jersey 07974
}

(Received 4 June 2003; accepted 17 February 2004; published 12 April 2004)

\begin{abstract}
In recent work [K. Srinivasan, P. E. Barclay, O. Painter, J. Chen, A. Y. Cho, and C. Gmachl, Appl. Phys. Lett. 83, 1915 (2003)] resonant mode linewidths of $0.10 \mathrm{~nm}$ (corresponding to a quality factor $\sim 1.3 \times 10^{4}$ ) were measured in a photonic crystal defect microcavity fabricated in an InAsP/ InGaAsP multi-quantum-well membrane. The quality of device fabrication is of critical importance in the performance of these devices. Here, we present the results of key processing steps, including inductively coupled plasma reactive ion etching of a $\mathrm{SiO}_{2}$ mask and the InAsP/InGaAsP membrane, and a selective undercut wet etch of an underlying sacrificial InP layer to create the freestanding membrane. The importance of etching through the membrane layer deeply into the sacrificial InP layer is highlighted, and discussed in the context of the crystallographic nature of the undercut wet etch process. The results of device processing are compared with previous work done using a chemically assisted ion-beam etch, and a discussion of the benefits of the current approach is given.

(C) 2004 American Vacuum Society. [DOI: 10.1116/1.1701848]
\end{abstract}

\section{INTRODUCTION}

Planar photonic crystal (PC) defect microcavities, which consist of an intentionally introduced deviation in the regularity of a two-dimensional periodic dielectric lattice, have attracted widespread attention due to a number of factors. Planar lithography, the process by which these devices are created, is both scalable and technologically mature. Using planar lithography, simple adjustments to the photonic lattice can be used to control the polarization, radiation direction, wavelength, and field profile of the cavity modes. ${ }^{1}$ From the standpoint of microcavity physics, PC microcavities are of interest because they can trap light to extremely small modal volumes, approaching the theoretical limit of a cubic halfwavelength in the material, ${ }^{2}$ and have been predicted to exhibit a quality factor $(Q)$ well in excess of $10000 .^{3-7}$

In a recent article, ${ }^{8}$ graded square lattice PC defect cavities with theoretically predicted $Q$ values of $10^{5}$ were fabricated in an InAsP/InGaAsP multi-quantum-well (MQW) membrane, and exhibited resonant modes with subthreshold linewidths of $0.10 \mathrm{~nm}$, corresponding to $Q \sim 1.3 \times 10^{4}$. The primary focus in Ref. 8 was on the measurement of these devices, including the $L-L$ (light in versus light out) curves of the lasing devices, subthreshold measurements of the resonance linewidth, and mode localization and polarization measurements that positively identify the mode as that studied in simulation. In this article, we focus on some aspects of the device fabrication that critically affect the cavity performance. In Sec. II, a brief description of the cavity design and the results of finite-difference time-domain (FDTD) simulations of the cavity mode of interest are given, drawing heavily upon original work ${ }^{5}$ in which the cavity was de-

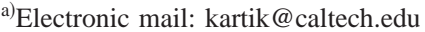

signed. Section III is the primary focus of this article, and is divided into subsection III A on mask creation and subsection III B on the high-temperature InAsP/InGaAsP membrane etch and subsequent $\mathrm{HCl}$ undercut wet etch of an underlying sacrifical InP layer. Of particular importance here is the relationship between the minimum hole radius that can be undercut and the etch depth through the membrane layer into the sacrifical layer, and how this in turn affects the $Q$ factor. Comparisons are made to previous fabrication processes used in the creation of PC membrane resonators ${ }^{9}$ to help elucidate some of the benefits of the current work. Finally, in Sec. IV, a brief summary of some of the measurements obtained from the PC cavities is given.

\section{CAVITY DESIGN}

The cavity geometry employed is shown in Fig. 1(a), and was designed using a combination of symmetry-based and Fourier space analyses, and FDTD simulations. ${ }^{5}$ The FDTDcalculated magnetic field amplitude and Fourier transformed dominant electric field component for the donor mode of interest in this cavity are shown in Figs. 1(b) and 1(c). FDTD results predict $Q \sim 10^{5}$ and a modal volume $V_{\text {eff }}$ $\sim 1.2(\lambda / n)^{3}$, where $n=3.4$ is the material refractive index.

Perhaps the most important feature of this graded square lattice design is its robustness. By utilizing symmetry to inherently suppress vertical radiation, and by employing a local perturbation in addition to a grade in the lattice filling fraction, the cavity $Q$ is relatively insensitive to perturbations in the size, shape, and position of individual holes (the sidewall angle is still problematic). More quantitatively, FDTD calculations show that the grade used in Fig. 1(a) can be varied significantly without reducing the $Q$ to a value less than $\sim 2 \times 10^{4}$. The robustness of the design is of particular 


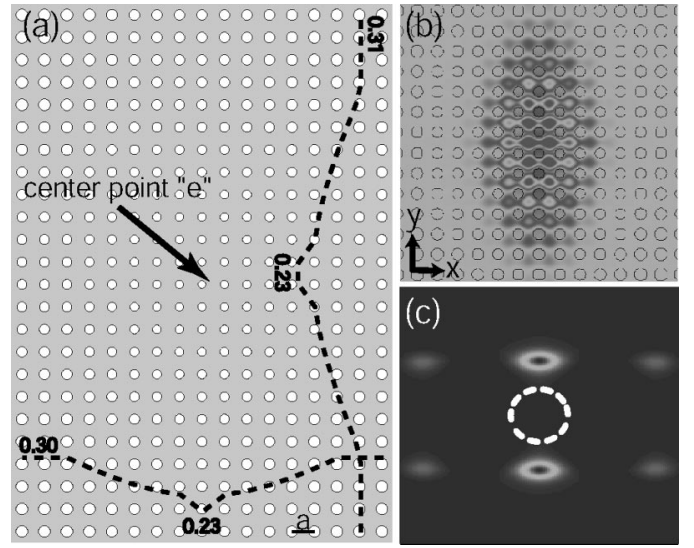

FIG. 1. (a) Graded square lattice cavity; dotted lines show the grade in hole radius ( $r / a)$ along the central $\hat{x}$ and $\hat{y}$ axes of the cavity. (b) Magnetic field amplitude $(|\mathbf{B}|)$ in the center of the PC membrane. (c) Fourier transformed dominant electric field component $\left(E_{x}\right)$. The dashed circle in (c) denotes the cladding light cone, showing that vertical radiation has been significantly suppressed. The normalized frequency of the mode is $a / \lambda_{0}=0.25$.

significance given the variations in hole shape and size that can occur in fabrication.

\section{DEVICE FABRICATION}

Devices are fabricated in a materials system with an active layer of five compressively strained InAsP QWs separated by InGaAsP barriers, as shown in Table I. The peak emission wavelength is at $\lambda=1284 \mathrm{~nm}$, and the epitaxy is designed so that, upon undercut of the $1.5 \mu \mathrm{m}$ sacrificial InP layer, a 252-nm-thick freestanding membrane will be formed (the thickness is chosen to be consistent with the emission wavelength of the QWs and the FDTD-calculated frequency of the cavity mode). The growth is carried out by solidsource molecular-beam epitaxy, as described elsewhere. ${ }^{10}$

\section{A. Mask creation}

A 200-nm-thick $\mathrm{SiO}_{2}$ mask layer is deposited on the epitaxial wafer by an Oxford Instruments Plasma Technology (OIPT) Plasmalab 100 plasma-enhanced chemical vapor deposition tool. The PC patterns are created through electron-beam (e-beam) lithography of an $\sim 350 \mathrm{~nm}$ layer of resist, typically polymethylmethacrylate or Zeon Chemical's ZEP-520A. Each cavity consisted of a total of 32 rows and 25 columns of air holes, with a lattice spacing of $a=305$, 315,325 , or $335 \mathrm{~nm}$ (normalized frequency of $a / \lambda \sim 0.25$ at $\lambda \sim 1.3 \mu \mathrm{m}$ ), for total cavity dimensions on the order of $8 \times 11$ $\mu \mathrm{m}$. The designed grade produces holes with radii $r$ between 70 and $110 \mathrm{~nm}$.

To etch the $\mathrm{SiO}_{2}$ mask layer, an OIPT Plasmalab 100 inductively coupled plasma reactive ion etch (ICP/RIE) tool with a $\mathrm{C}_{4} \mathrm{~F}_{8}$ gas chemistry was used. The requirements of the process were that the etched sidewalls be as smooth and vertical in slope as possible. Due to the relatively poor dry etch resistance of the e-beam resists, a third requirement was to avoid excessive damage to the resist, so that the desired shape and size of the holes remained intact. Along with this, the low etch selectivity of the resist $(\sim 1: 1)$ placed an upper limit on the thickness of the oxide layer used.

The etch profile and sidewall roughness were examined as a function of ICP power (400-600 W), rf power (50-100 $\mathrm{W}$ ), and $\mathrm{C}_{4} \mathrm{~F}_{8}: \mathrm{O}_{2}$ gas chemistry (between 20:0 sccm to 15:5 sccm), keeping a fixed chamber pressure ( $\left.P_{\mathrm{ch}}=6 \mathrm{mTorr}\right)$, lower electrode temperature $\left(\sim 20^{\circ} \mathrm{C}\right)$, and $\mathrm{He}$ backside cooling (20 Torr, $\sim 20 \mathrm{sccm}$ ). To maintain the integrity of the ICP/RIE chamber, $\mathrm{O}_{2}$ plasma cleans were periodically run between etches. For the process conditions examined, we did not observe significant changes in the sidewall roughness. For a given set of rf and ICP powers, we saw an increase in the sidewall verticality with increased $\mathrm{C}_{4} \mathrm{~F}_{8}$ content, without any polymerization, so that a pure $\mathrm{C}_{4} \mathrm{~F}_{8}$ gas chemistry was finally chosen. The rf and ICP powers were then adjusted to reduce damage to the resist as much as possible without causing degradation of the sidewall profile. For the final process conditions chosen $(\mathrm{rf}=80 \mathrm{~W}, \mathrm{ICP}=600 \mathrm{~W}, 20 \mathrm{sccm}$ $\mathrm{C}_{4} \mathrm{~F}_{8}$ ), an etch time of $\sim 2 \mathrm{~min}$ etched through the oxide layer completely and produced a sidewall angle of $\sim 85^{\circ}$, as estimated through cross-sectional scanning electron microscope (SEM) images. A SEM image of the etched $\mathrm{SiO}_{2}$ mask

TABLE I. Epitaxy for $1.3 \mu \mathrm{m}$ membrane lasers. $1.12 Q$ stands for quaternary GaInAsP layers, lattice matched to InP, with photoluminescence peak at $1.12 \mu \mathrm{m}\left(\mathrm{Ga}_{0.15} \mathrm{In}_{0.85} \mathrm{As}_{0.32} \mathrm{P}_{0.68}\right)$. The QW layers are $\operatorname{InAs}{ }_{0.48} \mathrm{P}_{0.52}$ and the barrier layers are $\mathrm{Ga}_{0.24} \mathrm{In}_{0.76} \mathrm{As}_{0.32} \mathrm{P}_{0.68}$. Total membrane thickness after undercutting sacrifical InP layer is 252 $\mathrm{nm}$.

\begin{tabular}{lcccc}
\hline \hline Layer & Materials & Strain (rel. to InP) & Thickness $(\AA)$ & Doping $\left(\mathrm{cm}^{-3}\right)$ \\
\hline $\begin{array}{l}\text { Separate } \\
\text { confinement }\end{array}$ & $1.12 Q$ & Unstrained & 810 & Undoped \\
Half-barrier & GaInAsP & $0.65 \%$ tens. & 60 & Undoped \\
$\begin{array}{l}\text { Active } \\
\text { region }\end{array}$ & 5 wells & $1.5 \%$ comp. & 60 & Undoped \\
Half-barrier & 4 barriers & $0.65 \%$ tens. & 120 & Undoped \\
Separate & GaInAsP & $0.65 \%$ tens. & 60 & Undoped \\
confinement & $1.12 Q$ & Unstrained & 810 & Undoped \\
Sacrificial buffer & InP & Unstrained & 15000 & Undoped \\
layer & & & & \\
Etch stop & InGaAs & Unstrained & 200 & Undoped \\
Substrate & InP & Unstrained & N/A & N/A \\
\hline \hline
\end{tabular}




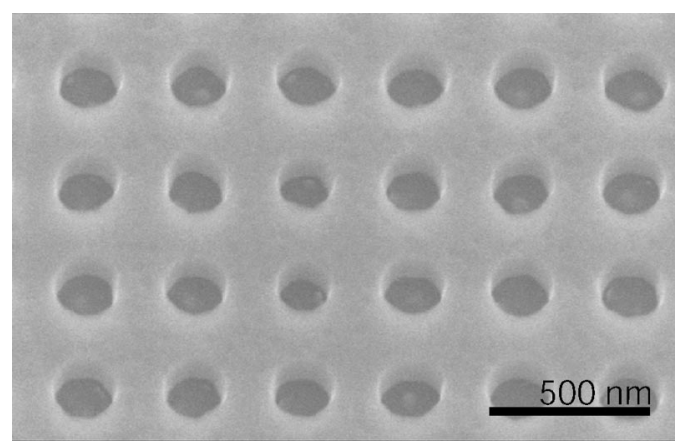

FIG. 2. Angled-view SEM image of the $\mathrm{SiO}_{2}$ etch mask.

layer is shown in Fig. 2, where an $\mathrm{O}_{2}$ plasma has been used to remove the remnants of the resist.

\section{B. InAsP/InGaAsP membrane etch and undercut}

The next step is an ICP/RIE etch through the InAsP/ InGaAsP membrane layer into the InP sacrifical layer. From the standpoint of cavity $Q$, it is of critical importance that the etched sidewalls be both smooth and vertical, as sidewall roughness will produce scattering loss, and an angled sidewall will break the vertical symmetry of the slab and will cause radiative coupling to modes of the opposite symmetry (TM-like modes). ${ }^{11}$ As we shall discuss, the requirements of the ICP/RIE etch are also influenced by the ensuing undercut wet etch, as the necessity to undercut all of the holes in the lattice places a lower limit on the etch depth into the InP layer.

Dry etching of In-containing III-V semiconductors is typically accomplished using one of two gas chemistries. ${ }^{12}$ The first, a $\mathrm{CH}_{4} / \mathrm{H}_{2}$ chemistry, is done at room temperature and can produce smooth etched morphologies, but with the drawbacks of a relatively slow etch rate $(\$ 60 \mathrm{~nm} / \mathrm{min})$ and heavy polymer deposition during the process. $\mathrm{Cl}_{2}$-based plasmas have also been used, but the low volatility of $\mathrm{InCl}_{x}$ products at room temperature necessitates some form of heating during the etch. This has been done in the past, using a highdensity ICP-produced $\mathrm{Cl}_{2}$ plasma, by Fujiwara et al., ${ }^{13}$ where the production of smooth etched surfaces is most likely due to a combination of the plasma providing local surface heating of the sample and an increased efficiency in the sputter desorption of the $\mathrm{InCl}_{x}$ products. ${ }^{12}$ Alternately, a number of studies have used a heated wafer table $\left(\gtrsim 150^{\circ} \mathrm{C}\right)$ with an $\mathrm{Ar}-\mathrm{Cl}_{2}$ chemistry to achieve a volatility of the $\mathrm{InCl}_{x}$ products sufficient to etch InP-related compounds with vertical sidewalls and smooth surface morphologies. In a recent study, ${ }^{14}$ Rommel and his collaborators optimize this etch (using $\mathrm{H}_{2}$ to control the sidewall profile) in an ICP/RIE system to produce submicron-width racetrack resonators with a $Q$ of 8000. We adopt an etch similar to the process used in that work. An important difference between this work and much of the published work on InP etching is the geometry we are etching, as the small features $(\sim 150 \mathrm{~nm}$ diameter holes) likely inhibit both the flow of ionized source gases to the etching surfaces and the removal of etched by-products. This
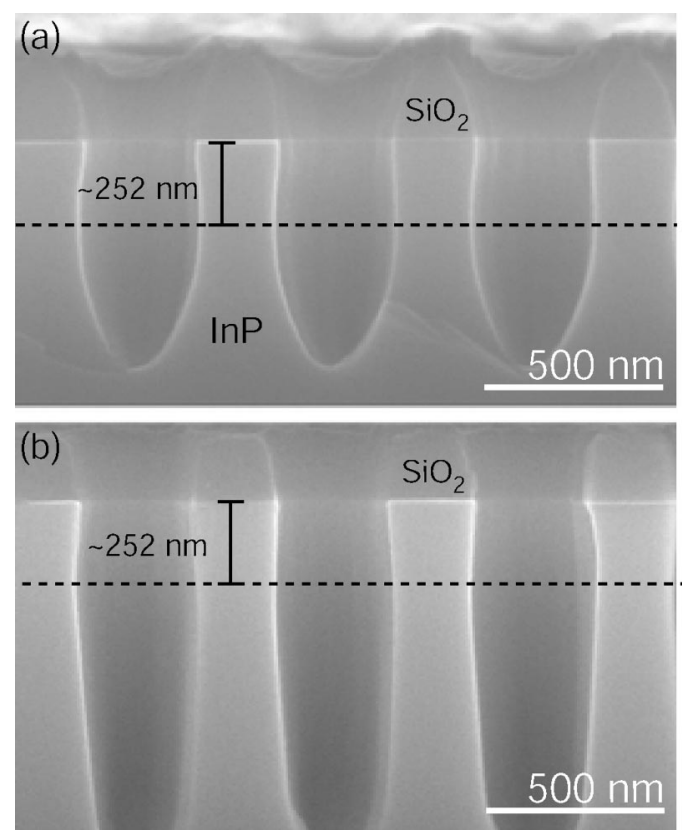

FIG. 3. SEM cross-sectional images of the InP etch for increasing wafer table temperature: (a) $T=150{ }^{\circ} \mathrm{C}, t_{\mathrm{etch}}=120 \mathrm{~s}$, etch depth $=650 \mathrm{~nm}$, and (b) $T=180^{\circ} \mathrm{C}, t_{\text {etch }}=165 \mathrm{~s}$, etch depth $=1 \mu \mathrm{m}$. For these etches, $\mathrm{rf}=100 \mathrm{~W}$, $\mathrm{ICP}=300 \mathrm{~W}, P_{\mathrm{ch}}=2 \mathrm{mTorr}$, and $\mathrm{Ar}_{\mathrm{Cl}_{2}}=12: 3 \mathrm{sccm}$. The top $252 \mathrm{~nm}$, highlighted in each SEM image, corresponds to the active waveguiding layer thickness (final membrane thickness) in the actual InAsP/InGaAsP laser material used in the device fabrication.

will affect both the etch rates we observe (often a factor of 2 or more lower than the etch rates in open areas) and the resulting surface morphology.

We examined etched samples (initially etching pure InP rather than the InAsP/InGaAsP QW material) primarily as a function of gas composition ( $\mathrm{Ar}: \mathrm{Cl}_{2}$ ratio between 9:6 sccm and $13: 2 \mathrm{sccm})$, stage temperature $\left(20-225^{\circ} \mathrm{C}\right)$, rf power (100-200 W), and ICP power $(250-400 \mathrm{~W})$. A preliminary etch with a stage temperature of $150{ }^{\circ} \mathrm{C}$ and an $\mathrm{Ar}: \mathrm{Cl}_{2}$ ratio of 12:3 sccm is shown in Fig. 3(a). By further increasing the stage temperature [Fig. 3(b)], the profile of the holes becomes more vertical and the total etch rate increases. We chose a stage temperature of $205^{\circ} \mathrm{C}$ as temperatures above this value provided no added benefit to the sidewall smoothness and profile of the etched holes. With a stage temperature of $205^{\circ} \mathrm{C}$, the $\mathrm{Ar}: \mathrm{Cl}_{2}$ gas ratio was varied around $12: 3 \mathrm{sccm}$. It was found that higher concentrations of $\mathrm{Cl}_{2}$ produce a slight amount of sidewall roughness, while lower concentrations produce a more pronounced overcut etch profile. With a stage temperature of $205^{\circ} \mathrm{C}$ and an $\mathrm{Ar}: \mathrm{Cl}_{2}$ gas ratio of $12: 3$ $\mathrm{sccm}$, the ICP and rf powers were then varied about values of 300 and $100 \mathrm{~W}$, respectively. Larger rf powers caused deterioration of the oxide mask, producing some sidewall erosion, while smaller rf powers reduced the etch anisotropy. We noticed little variation in etch quality as a function of ICP power over the range of ICP powers explored. The final InGaAsP material etch conditions that we settled on were: $T$ $=205^{\circ} \mathrm{C}, \mathrm{Ar}: \mathrm{Cl}_{2}=12: 3 \mathrm{sccm}, P_{\mathrm{ch}}=2 \mathrm{mTorr}, \mathrm{rf}=100 \mathrm{~W}$, and $\mathrm{ICP}=300 \mathrm{~W}$. With these process conditions and an etch 

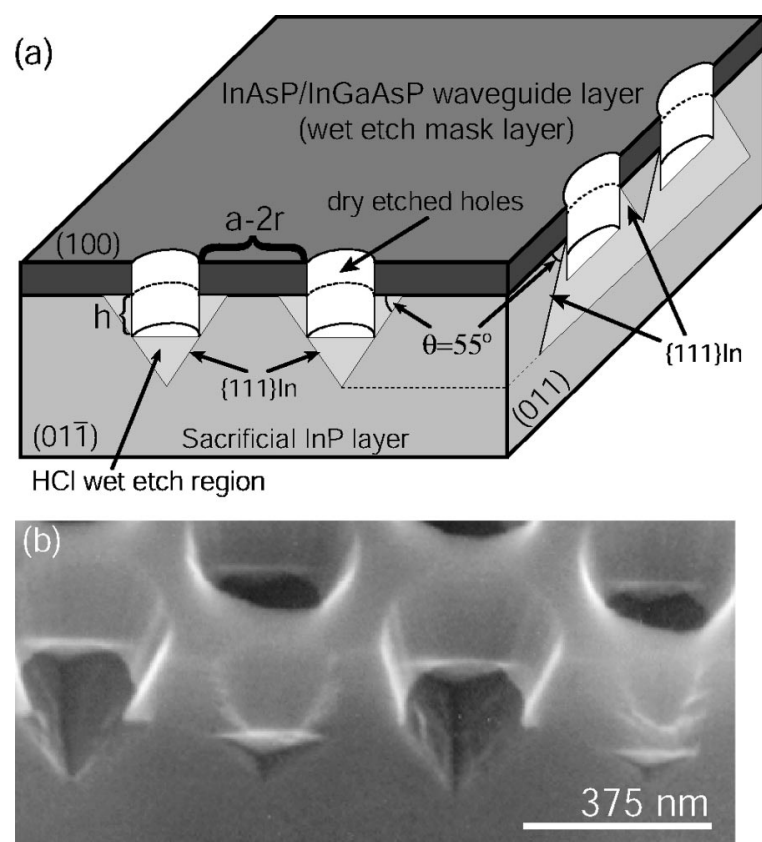

FIG. 4. (a) Illustration of the shape of the etched surfaces resulting from the crystallographic nature of the InP wet etch $(h=$ etch depth below waveguiding layer into InP sacrificial layer, $r=$ hole radius, $a=$ lattice spacing, $(a$ $-2 r)=$ gap between holes). (b) SEM image of a partially undercut membrane (where the membrane layer was etched with a CAIBE system) showing the $\{111\}_{\text {In }}$ crystal plane facets. Both figures adapted from previous work (see Ref. 9).

time of $120 \mathrm{~s}$, an etch depth of $1.5 \mu \mathrm{m}$ was achieved, with a selectivity of $\sim 20: 1$ to the oxide mask.

To remove the sacrificial InP layer and undercut the membrane, an $\mathrm{HCl}: \mathrm{H}_{2} \mathrm{O}(4: 1)$ solution was used. $\mathrm{HCl}$ etches the InP layer at a much faster rate than it does the active waveguide layer [composed of lower phosphorous $(\mathrm{P})$ percentage alloys of InAsP and InGaAsP compounds], providing the necessary degree of etch selectivity. ${ }^{15}$ The resolution and selectivity of the etch are improved by cooling the solution in an ice-water bath to $\sim 4{ }^{\circ} \mathrm{C}$, thereby reducing the material etch rates and, in particular, the total amount of waveguide material that is necessarily etched. The typical time to fully undercut the membrane was $\sim 12$ min (depending on the lattice spacing and hole size of the PC pattern). To protect the top sample surface, the remnants of the oxide mask layer were removed only after the undercut etch was performed.

The $\mathrm{HCl}: \mathrm{H}_{2} \mathrm{O}$ solution etches InP with a strong crystallographic dependence. ${ }^{16}$ The resulting etch shape is determined by the differences in etch rate of different crystallographic planes as well as boundary conditions imposed by masking layers (which limit the available etch angles). The etch shape will ultimately converge to one determined by the slowest set of etch planes, which are the indium (In)-rich $\{111\}$ planes $\left(\{111\}_{\text {In }}\right)$ for the InGaAsP material system. For an (100) InP wafer, the $\{111\}_{\text {In }}$ planes are at an angle of $\sim 55^{\circ}$ from the surface normal as shown in Fig. 4(a). For concave mask openings, this results in an etch cross section which is an overcut $\mathrm{V}$ shape in the $(01 \overline{1})$ plane and an undercut overhanging shape in the $(01 \overline{1})$ plane. This is shown schemati-
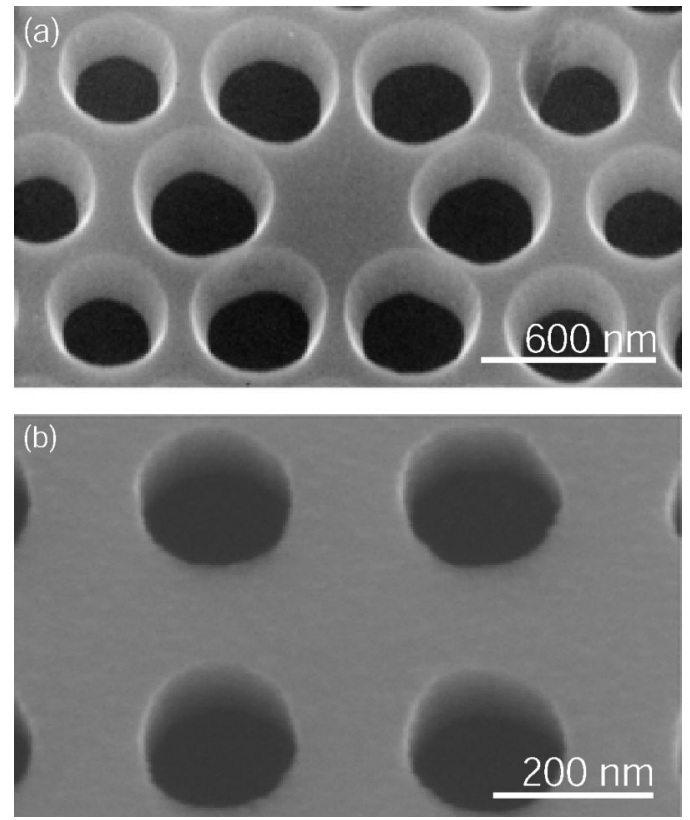

FIG. 5. SEM micrographs of fully fabricated devices. (a) Previous work using CAIBE for the membrane etch (see Ref. 9). (b) The current work, using ICP/RIE for the membrane etch.

cally in Fig. 4(a), and can also be seen clearly in the partially undercut holes of Fig. 4(b). As in Fig. 4(b), once the etch has proceeded to the $\{111\}_{\text {In }}$ planes, unless there is an overlap between the $\{111\}_{\text {In }}$ planes of adjacent holes, the etch will grind to a halt and the waveguiding layer will not completely undercut (longer etch times and/or higher temperatures may be employed to further undercut the structure, however, selectivity to the active waveguiding layer will have been lost). As a result, the ICP/RIE dry etch depth into the underlying sacrificial InP layer sets a lower limit on the radius $(r)$ and lattice spacing $(a)$ of holes that can be undercut. More precisely, using the nomenclature of Fig. 4(a), the adjacent $\{111\}_{\text {In }}$ planes in the $(01 \overline{1})$ plane overlap for $h \geqslant(a / 2$ $-r) \tan \left(55^{\circ}\right)$, so that $h$ increases as $r$ decreases for a given $a$. This was a limitation of previous work done using an $\mathrm{Ar}: \mathrm{Cl}_{2}$ chemically assisted ion-beam etch (CAIBE) ${ }^{9}$ for which total etch depths were typically $\sim 340 \mathrm{~nm}$, corresponding to $h$ $\sim 130 \mathrm{~nm}$. For the fabricated lattice spacings in that work $(a=500 \mathrm{~nm})$, this produces a minimum possible undercut hole radius of $r / a=0.32$. The ability to undercut smaller holes (preferably as small as $r / a=0.20$ ) is of critical importance to the cavity $Q$, as holes that are too large in size will significantly raise the frequency of a given mode and hence the size of the cladding light cone, thereby increasing the amount of power radiating vertically. ${ }^{5}$ Our etch depth of $\sim 1.5 \mu \mathrm{m}(h \sim 1.25 \mu \mathrm{m})$ is not only deep enough to ensure that the $\{111\}_{\text {In }}$ crystal planes overlap for even the smallest desired holes $(r / a=0.23)$, but also aids the undercut process by reducing the total amount of material that needs to be removed.

Figure 5 compares the results of previous fabrication methods ${ }^{9}$ with the current work. Both images show smooth membrane sidewalls, but the sidewall profile is considerably 

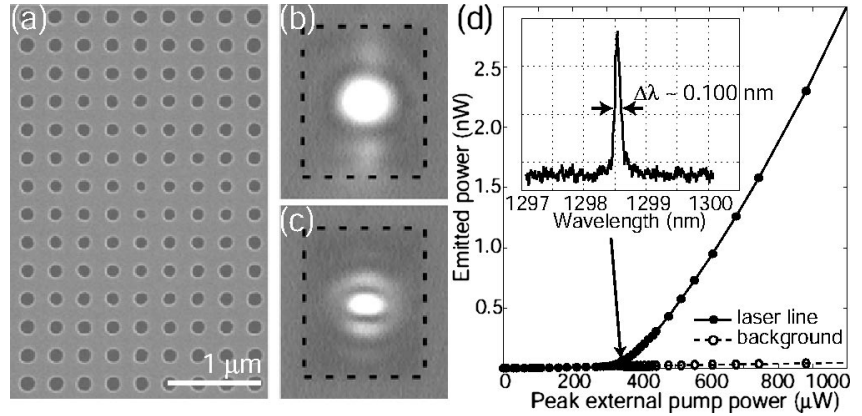

FIG. 6. (a) SEM image of a graded lattice PC microcavity in InAsP MQW material (lattice constant $a \sim 305 \mathrm{~nm}$ ). Optical images of a cavity pumped with a (b) broad pump beam and (c) focused pump beam (dashed lines indicate cavity boundary). (d) $L-L$ curve and subthreshold spectrum (inset) of a graded square lattice PC microcavity pumped with a spatially broad pump beam (10 ns pulse, $300 \mathrm{~ns}$ period).

more sloped in the former work [this is also evident in Fig. 4(b)]. The higher degree of verticality and the ability to undercut the smallest desired holes $(r / a=0.23)$ are the primary advantages of the present work. Although optimization of all of the masking steps was necessary to achieve this result, the ICP/RIE membrane etch is the critically important step that creates these advantages.

\section{MEASUREMENTS}

Lasing data and subthreshold cavity linewidth measurements of the fabricated devices are described in detail in a recent article. ${ }^{8}$ We briefly summarize some of the important results of that work here.

In Fig. 6(a), we show a SEM micrograph of a fully processed device. Devices are optically pumped (typically with a $10 \mathrm{~ns}$ pulse width and $300 \mathrm{~ns}$ period) at room temperature with a semiconductor laser at $830 \mathrm{~nm}$ through a $20 \times$ objective lens (numerical aperture $=0.4$ ), also used to collect emitted photoluminescence (PL) into an optical spectrum analyzer. The observed lasing threshold is critically dependent on the characteristics of the pumping beam. For linewidth measurements, we pump the cavity with a fairly broad beam [Fig. 6(b), area $\sim 21 \mu^{2}$ ] for two reasons: (i) the broad pump beam covers a significant portion of the cavity area, so that after diffusion of carriers, the majority of the cavity should be pumped and therefore nonabsorbing; and (ii) use of a broad pump beam limits the effects of thermal broadening. A typical $L-L$ curve using the broad pump beam condition is shown in Fig. 6(d), where the power in the laser line is integrated over a $10 \mathrm{~nm}$ bandwidth about the laser wavelength of $\lambda=1298.5 \mathrm{~nm}$. As discussed in Ref. 8, an estimated laser threshold of $360 \mu \mathrm{W}$ is determined both by extrapolating back from the $L-L$ curve above threshold and by examining a slope change in the background off-resonance emission. The linewidth at our estimate of transparency pump power is $\Delta \lambda=0.10 \mathrm{~nm}$, corresponding to $Q \sim 1.3 \times 10^{4}$, our best estimate of the cold cavity $Q$ [Fig. 6(d), inset]. In addition, by using a more tightly focused beam [Fig. 6(c)], the lasing threshold is considerably reduced to external pump power levels as low as $\sim 100 \mu \mathrm{W}$. Measurements of the laser mode polarization and the localization of the cavity mode were also made and were consistent with theoretical predictions.

In summary, we have described the fabrication process recently used to create photonic crystal microcavities in an InAsP/InGaAsP MQW membrane with subthreshold linewidths of $\Delta \lambda=0.10 \mathrm{~nm}$, corresponding to a cavity $Q$ of 1.3 $\times 10^{4}$. The primary processing steps of note are electronbeam lithography, mask transfer into $\mathrm{SiO}_{2}$, a deep $(\sim 1.5$ $\mu \mathrm{m})$ etch through the membrane layer into a sacrifical InP layer, and an undercut wet etch to create the freestanding membrane. The dielectric mask and semiconductor waveguide are both etched using an ICP/RIE tool, where the independent control of plasma density (through the ICP source) and the kinetic energy of the ionized gases (through control of the lower electrode) combined with a variety of available source gases and the ability to heat the wafer table allow for a number of different materials systems to be etched with smooth, vertical sidewalls. The ability to deeply etch through the membrane into the sacrifical InP layer allows holes of small radius to be completely undercut, a limitation that existed in previous work. This ability, along with improvements in the verticality of the etched sidewalls, are essential to creating high- $Q$ PC membrane microcavities.

\section{ACKNOWLEDGMENTS}

One of the authors (K. S.) thanks the Hertz Foundation for its financial support. The authors thank Alan Leung from OIPT and Thomas Johnson from Caltech for support with the ICP/RIE tool.

${ }^{1}$ O. Painter, K. Srinivasan, J. D. O'Brien, A. Scherer, and P. D. Dapkus, J. Opt. A, 3, S161 (2001).

${ }^{2}$ O. Painter, R. K. Lee, A. Yariv, A. Scherer, J. D. O'Brien, P. D. Dapkus, and I. Kim, Science 284, 1819 (1999).

${ }^{3}$ O. Painter, J. Vučković, and A. Scherer, J. Opt. Soc. Am. B 16, 275 (1999).

${ }^{4}$ J. Vučković, M. Lončar, H. Mabuchi, and A. Scherer, Phys. Rev. E 65, (2002).

${ }^{5}$ K. Srinivasan and O. Painter, Opt. Express 10, 670 (2002).

${ }^{6}$ K. Srinivasan and O. Painter, Opt. Express 11, 579 (2003).

${ }^{7}$ H.-Y. Ryu, M. Notomi, and Y.-H. Lee, Appl. Phys. Lett. 83, 4294 (2003).

${ }^{8}$ K. Srinivasan, P. E. Barclay, O. Painter, J. Chen, A. Y. Cho, and C. Gmachl, Appl. Phys. Lett. 83, 1915 (2003).

${ }^{9}$ O. J. Painter, Ph.D. thesis, California Institute of Technology, 2001.

${ }^{10}$ W.-Y. Hwang, J. Baillargeon, S. N. G. Chu, P. F. Sciortino, and A. Y. Cho, J. Vac. Sci. Technol. B 16, 1422 (1998).

${ }^{11}$ Y. Tanaka, T. Asano, Y. Akahane, B.-S. Song, and S. Noda, Appl. Phys. Lett. 82, 1661 (2003).

${ }^{12} \mathrm{C}$. Youtsey and I. Adesida, Dry Etching of InP and Related Materials, in Handbook of Advanced Plasma Processing Techniques (Springer, Berlin, 2000), pp. 479-483.

${ }^{13}$ Y. Fujiwara, K. Kikuchi, M. Hashimoto, H. Hatate, T. Imai, Y. Takeda, H. Nakano, M. Honda, T. Tatsuta, and O. Tsujii, Jpn. J. Appl. Phys. 36, 7763 (1997).

${ }^{14}$ S. L. Rommel, J.-H. Jang, W. Lu, G. Cueva, L. Zhou, G. Pajer, R. Whaley, A. Lepore, Z. Schellanbarger, and J. H. Abeles, J. Vac. Sci. Technol. B 20, 1327 (2002).

${ }^{15}$ P. Mounaix, P. Delobelle, X. Mélique, L. Bornier, and D. Lippens, Mater. Sci. Eng., B 51, 258 (1998).

${ }^{16} \mathrm{~S}$. Adachi and H. Kawaguchi, J. Electrochem. Soc. 128, 1342 (1981). 